\title{
Cortical regions activated by the subjective sense of perceptual coherence of environmental sounds: A proposal for a neuroscience of intuition
}

\author{
KIRSTEN G. Volz \\ Max Planck Institute for Neurological Research, Cologne, Germany \\ and Max Planck Institute for Human Cognitive and Brain Sciences, Leipzig, Germany \\ RUDOLF RÜBSAMEN \\ University of Leipzig, Leipzig, Germany \\ AND \\ D. Yves von Cramon \\ Max Planck Institute for Neurological Research, Cologne, Germany \\ and Max Planck Institute for Human Cognitive and Brain Sciences, Leipzig, Germany
}

\begin{abstract}
According to the Oxford English Dictionary, intuition is "the ability to understand or know something immediately, without conscious reasoning." In other words, people continuously, without conscious attention, recognize patterns in the stream of sensations that impinge upon them. The result is a vague perception of coherence, which subsequently biases thought and behavior accordingly. Within the visual domain, research using paradigms with difficult recognition has suggested that the orbitofrontal cortex (OFC) serves as a fast detector and predictor of potential content that utilizes coarse facets of the input. To investigate whether the OFC is crucial in biasing task-specific processing, and hence subserves intuitive judgments in various modalities, we used a difficult-recognition paradigm in the auditory domain. Participants were presented with short sequences of distorted, nonverbal, environmental sounds and had to perform a sound categorization task. Imaging results revealed rostral medial OFC activation for such auditory intuitive coherence judgments. By means of a conjunction analysis between the present results and those from a previous study on visual intuitive coherence judgments, the rostral medial OFC was shown to be activated via both modalities. We conclude that rostral OFC activation during intuitive coherence judgments subserves the detection of potential content on the basis of only coarse facets of the input.
\end{abstract}

In everyday life, we all make decisions nonconsciously, without carefully examining and integrating each bit of information. Rather, we rely on a feeling of knowing what decision to make, especially in the presence of uncertainty, perhaps due to time pressure or the importance of gauging the probability of consequences. These types of spontaneous judgment processes are collectively defined as intuition (see, e.g., Hogarth, 2001; Kahneman \& Frederick, 2002). The process of intuition is hence characterized by a lack of awareness of how the outcome has been achieved (Hogarth, 2001). In this context, it is important to consider two issues: First, the concept of intuition means different things to different people and has attracted the attention of several schools and scholars. Second, the term intuition appears "intuitively clear," yet a concise, generally accepted definition has not yet been provided in the scientific literature (see Hogarth, 2001). Typically, intuition is defined negatively, by contrasting it with analysis and logical thought - that is, intuition is regarded as a cognitive process that somehow produces an answer, solution, or idea without consciously going through a logically defensible step-by-step process (Hammond, 2007). Yet, such definitions leave room for several contrary assumptions about the nature of intuitive processes. Alternatively, some researchers have proposed lists of the features of intuition, including such attributes as holistic, associative, fast, and automatic (Epstein, 1973; Hammond, Hamm, Grassia, \& Pearson, 1987). However, these listings leave open the question of how many attributes are necessary for the recognition of intuitive processes. For some time, the literature on human judgment and decisionmaking eluded the problem of defining intuition by linking it almost exclusively to some well-defined simplifying judgment heuristics (for an overview, see Gilovich, Griffin, \& Kahneman, 2002; Kahneman, Slovic, \& Tversky, 1982). In recent years, another view has been gaining influence in the field of judgment and decision-making, termed the 
learning perspective (Hogarth, 2001; Plessner, Betsch, \& Betsch, 2008). According to this view, intuition relies on mental representations that reflect the entire stream of prior experiences; it is assumed to capitalize on these stored representations to often provide direct access to the criterion to be judged.

Because the term intuition has multiple connotations and is used with an "intuitive" understanding, in the context of the present article it is important that we specify our understanding of the term's meaning and of the nature of intuitive processes: In accordance with the learning perspective, we assume intuitions to be difficult-toarticulate, affect-laden recognitions or judgments, based on prior learning and experience, that are arrived at through holistic associations (see Sadler-Smith, 2008). From an information-processing perspective (and following the literature on priming), it has been suggested that specific situational cues automatically activate, probably by a process of spreading activation (see Anderson, 1983), a mnemonic network that integrates the entire stream of prior experiences that are critically related to an event. This mnemonic network is also denoted as a chunk. Because of its associative preactivation, the chunk as a whole is processed more fluently than expected, presumably because the chunk's constituents mutually facilitate their processing. This fluency is assumed to impinge on the current affective state in a positive way. Subsequently, the fluency-triggered affect may be used in explicit judgments. Crucially, it is not the fluency per se, but rather the affective derivatives of processing fluency, that can enter awareness and serve as a basis for deliberative judgments (see Reber, Wurtz, \& Zimmermann, 2004).

As examples of experimentally testable intuitions defined in this way, consider judgments of coherence (semantic or perceptual) that require spontaneous guesses about whether things fit or are "out of kilter" (see Bowers, Regehr, Balthazard, \& Parker, 1990; Ilg et al., 2007; Topolinski \& Strack, 2008). By using a semantic coherence task, Topolinski and Strack $(2007,2008)$ have shown that (1) coherent word triads, which relate to a common remote associate, are processed more fluently than noncoherent word triads, which lack such an associate; (2) the high fluency of the coherent triads triggers a positive affect; and (3) the mere visual presentation of a coherent triad automatically facilitates subsequent processing of the triad's common remote associate. These findings support the assumption that we have outlined: In the case of coherent word triads, convergent associative spreading may bring about partial activation of a searched-for common remote associate, which is embodied in a "gut" feeling.

Individuals are able to efficiently judge not only the coherence of word triads, but also the coherence of visual stimuli, in the form of "gestalt closure stimuli" (Waterloo gestalt closure task; Bowers et al., 1990). In the Waterloo gestalt closure task, distorted meaningful stimuli, consisting of common objects, are paired with stimuli in which the pixel information of the meaningful gestalts is dislocated into nonmeaningful patterns. Accordingly, both the coherent and noncoherent stimuli consist of identical pixel information, differing merely in the arrangement of the elements. As with the word triads, individuals in Bowers et al.'s study were able to detect the coherent gestalts, even if they were unable to express what those gestalts represented. These trials, on which participants exhibited a "feeling of warmth," were classified as representing intuitive judgment processes.

In a previous imaging study in which we aimed to identify the brain regions involved in intuitive judgments of visual coherence, we used a modified version of the Waterloo gestalt closure task. We presented participants with (individual) fragmented black-and-white line drawings of common objects; the participants had to indicate, in a forced choice procedure, whether or not the given stimulus was coherent (Volz \& von Cramon, 2006). The results revealed specific activation within the medial orbitofrontal cortex (OFC) for subjective coherence judgments, even when participants were unable to name the object concerned. That is, the subjective sensation that the presented stimulus was coherent was associated with OFC activation. In line with results from Bar et al. (2006), neuroanatomical data (see Kringelbach, 2005; Kringelbach \& Rolls, 2004), and animal studies (see Schoenbaum \& Roesch, 2005; Schoenbaum, Roesch, \& Stalnaker, 2006), our findings suggested that OFC activation serves as "a rapid detector and predictor of potential content based on coarse aspects of the input" (Bar et al., 2006, p. 453). The fact that the OFC in a demanding object recognition task peaks $50 \mathrm{msec}$ before the fusiform gyrus, an area known to be involved in object recognition processes, implies that $\mathrm{OFC}$ is used for extrapolation beyond the directly accessible sensory information (see Bar et al., 2006). Such coherence judgments are supposed to be characterized by immediacy, self-evidence, extrapolativeness, and coerciveness.

In the present study, we sought to test whether the OFC generally - that is, in various modalities - provides a predictive initial guess that biases subsequent thought and action. Accordingly, we adapted our paradigm for the auditory domain. Our participants heard either short sequences of nonverbal environmental sounds or backward versions of the same sounds, both superimposed with noise and/ or periods of zero sound energy. Hence, the backward versions matched the forward sounds on most acoustic features but differed in temporal sequence. That is, the temporal displacement of the elements of the meaningful sound (the "organized whole") resulted in a nonmeaningful pattern. While listening, participants performed a forced choice sound categorization task; that is, they had to indicate as fast as possible for each sound whether or not it was coherent, and hence whether it represented an actual auditory event. This ability to recognize different types of environmental (nonverbal) sounds is thought to derive, in part, from automatically abstracting the physical properties of a sound and matching them to the implicit known attributes of given sound categories (see, e.g., Medin \& Coley, 1998). Accordingly, coherence judgments are taken to reflect intuitive judgment processes.

The auditory nature of the stimuli here ruled out a very short presentation time, like the one implemented in our visual study (400-msec presentation of the stimuli), because sound only develops structure over time. Accordingly, in 
the present paradigm all of the sound stimuli were trimmed to $5 \mathrm{sec}$ in duration. This relatively long presentation time, however, could increase the likelihood of coherence judgments, which are not based on a feeling, but rather resemble fully formed judgments. That is, judgments for the forwardplayed sound stimuli correctly identified as coherent (hits) were likely to result from an easy-to-articulate recognition process rather than from recognition that appeared driven simply by a feeling. By definition, intuition draws on thinking processes that are largely nonconscious. Hence, it was not possible to ask participants about the nature of their judgments. Instead, we played sound stimuli backward, because their components still contained clues of coherence, which could activate a chunk. Accordingly, in the present sound recognition task, the backward-played sound stimuli incorrectly classified as coherent (false alarms [FAs]) were taken to reflect intuitive judgment processes. This was because the global composition of a backward-played sound stimulus exclusively distinguishes it as (objectively) noncoherent. Since the recognition process was here made difficult by the distortion manipulation, though, the assessment of coherence for these stimuli was likely to be based on parts of the stimulus sensed to be coherent rather than on the overall arrangement. Accordingly, especially for FAs, we expected activation within medial OFC with the backward-played stimuli.

After the fMRI session, we played back to the participants only those sound stimuli they had earlier classified as coherent, and they had to write down their ideas about what the stimuli represented. According to the fluency conception of intuitive processes that we have outlined, the repeated presentation of a stimulus previously classified as coherent should facilitate its recognition by means of confluent semantic spreading. Thus, the participants who came up with many ideas about the stimulus sources were considered to be more intuitive, so we expected this group to show enhanced OFC activation in the initial task, relative to those who provided relatively few ideas about what the auditory stimuli represented.

Since our area of interest (OFC) is known to be sensitive to susceptibility artifacts and geometric distortions when standard echo-planar imaging (EPI) is used, we used instead a spin-echo (SE) EPI sequence and a distortion correction procedure (according to the method described by Jezzard \& Balaban, 1995; see the Method section below for more details).

\section{METHOD}

\section{Participants}

A group of 14 right-handed, healthy volunteers ( 7 female; mean age 25.5 years, $S D 3.7$, range 19-31) with no history of neurological, psychiatric, or auditory symptoms participated in the fMRI experiment. Informed consent was obtained from each participant according to the declaration of Helsinki. The experimental standards were approved by the ethics committee of the University of Leipzig. All data were handled anonymously.

\section{Materials}

Stimuli, task, and experimental session. The experimental materials consisted of auditory stimuli presented binaurally and a visual reminder of the response key assignment. Participants placed the index and middle fingers of the right hand on the "left" and "right" response buttons, respectively. Within each trial, an auditory stimulus was presented for $5 \mathrm{sec}$, concurrently with the response key assignment. As soon as the participant pressed a response key, which was possible throughout the entire presentation of the auditory stimulus, the visual reminder turned red to indicate that the response had been recorded. Each auditory stimulus was played from start to finish, regardless of when the participant made a response. The next trial began after a mean interstimulus interval of $2 \mathrm{sec}$, during which a fixation cross was displayed.

The auditory stimuli were short sequences of nonverbal environmental sounds or temporally reversed (backward) versions of these same sounds, both superimposed with pink noise (i.e., essentially white noise, but with energy in inverse proportion to frequency and, hence, more energy in the bass than in the treble) and/or periods of zero sound energy. The sound samples were compiled from a CD collection of professionally recorded sounds (i.e., sound effects; Digimore Ltd.). Examples are the ringing of a church bell, the gurgle of a wastewater disposal line, or the rattling of cutlery (see the Appendix for a complete list). All sound stimuli were trimmed to $5 \mathrm{sec}$ in duration and temporally reversed for the backward presentation (using Audition software, version 1.5; Adobe Systems, Inc.). The temporally reversed sounds matched the physical features of the original sounds in overall intensity, duration, spectral content, spectral variation, and acoustic complexity. To make recognition more difficult, and thus to increase reliance on a feeling of coherence, $93 \%$ of the sound stimuli were distorted, as described now: First, $7 \%$ of the sound stimuli were superimposed with pink noise, which is similar to natural sounds, such as a waterfall. The level of the noise signal was specifically adjusted for each sound stimulus. Another $43 \%$ of the sound stimuli were distorted by reducing the sound energy of the signal to zero for a specific number of 200-msec periods (see below). Since this procedure results in an amplitude plot that looks like a picket fence, these were termed fence stimuli. Six types of fence stimuli were generated, which differed in the temporal arrangement of the breaks - that is, the length of the pickets was varied pseudorandomly, so that the signal was presented for $200,250,300,400,600$, or $800 \mathrm{msec}$. The remaining $43 \%$ of the sound stimuli were distorted by applying both the fence procedure and superimposed pink noise. In an initial pilot study, 8 participants (not included in the fMRI experiment) screened the forward- and backward-played sound samples, retaining 68 forward-played and 71 backward-played sound stimuli. In a second pilot study, another 8 participants (also not in the fMRI experiment) performed a forced choice sound categorization task. For a probability of hits of .59 and a probability of false alarms of .32, the discriminability index $d^{\prime}$ was .71 , indicating a relatively difficult categorization task. Furthermore, participants' response strategies were revealed to be conservative; that is, they responded "no" more often than an ideal observer would $(C=.11$; see Abdi, 2007). To foster uncertainty in the fMRI experiment, the difficulty of the paradigm was not changed, but to induce a more liberal response strategy (i.e., responding "yes" more often than an ideal observer), the participants were instructed to categorize sound stimuli as coherent whenever they could verbalize, describe, or visualize the likely sound source, or whenever they had implicit knowledge or a general sense of familiarity about the source. Hence, we emphasized that participants need not be able to name the specific stimulus in order to categorize it as coherent.

In the actual fMRI experiment, the participants were presented with 150 stimuli (68 forward-played sound stimuli, 71 backwardplayed sound stimuli, and 11 null events) in a pseudorandom order and were asked to perform a sound categorization task. The response assignments were balanced across participants. All trials lasted for $8 \mathrm{sec}$ - that is, four scans with a repetition time (TR) of $2 \mathrm{sec}$. To allow for measurements to be taken at numerous time points along the blood-oxygenation-level-dependent signal curve, the onset of each stimulus presentation relative to the beginning of the first of the three scans was varied randomly in four time steps $(0,500,1,000$, 
and $1,500 \mathrm{msec}$ ). The purpose of this procedure was to enhance the temporal resolution of the image acquisition (Birn, Cox, \& Bandettini, 2002; Miezin, Maccotta, Ollinger, Petersen, \& Buckner, 2000). Since recognition of backward sound trials would be facilitated by previous experience with the corresponding forward sound, and vice versa, a given sound stimulus was always presented in a different distortion version than during its preceding presentation. This procedure was possible because we produced 72 stimuli altogether, each in 14 variations. After the experimental session, those stimuli that participants had previously categorized as meaningful auditory objects were played back for them, and they were asked to describe each sound source. Subsequently, the participants filled out a questionnaire capturing their preference for intuition versus deliberation (Betsch, 2004), and they were thanked and debriefed.

\section{MRI Scanning Procedure}

Imaging was performed with a 3 T scanner (Medspec 30/100; Bruker Optics, Ettlingen, Germany). The participants wore custom earplugs (HearSafe Group, Cincinnati, Ohio) and were presented with binaural sound stimuli. To ensure that participants could easily hear the sound stimuli via custom headphones (Resonance Technology Inc., Northridge, California), the volume was individually adjusted before scanning. Since we expected activation within the $\mathrm{OFC}$, and this cortical area is known to be affected by strong susceptibility gradients, we used an SE sequence, which has been shown to be less sensitive to susceptibility-related signal dropout than are gradient-echo (GE) sequences (Norris, Zysset, Mildner, \& Wiggins, 2002; Schmidt, Boesiger, \& Ishai, 2005). The drawback of using SErather than GE-based fMRI, however, is the lower statistical power of SE sequences, because the advantage in sensitivity of the SE EPI is counteracted by the noise produced by switching the magnetic field gradients on and off. However, since task difficulty in terms of auditory perception was desired, this issue was not regarded as a major drawback, particularly because the noise affected all trials, and thus its effect was not condition-specific. Any geometric distortions due to static magnetic field inhomogeneities were characterized by a field-map scan at the end of each session. By use of the resulting field maps, a distortion correction was applied to the functional-imaging data via the method described by Jezzard and Balaban (1995), without intensity correction and with a nonlinear interpolation (i.e., an Akima spline). Seventeen axial slices (4-mm thickness, interslice gap $1 \mathrm{~mm}$, field of view $19.2 \mathrm{~cm}$, data matrix $64 \times 64$ voxels, and in-plane resolution $3 \times 3 \mathrm{~mm}$ ) parallel to the bicommissural plane (AC-PC) were acquired using an SE EPI sequence (TR $2 \mathrm{sec}$, echo time [TE] $75 \mathrm{msec}$ ). Because we used an SE sequence with a TR of $2 \mathrm{sec}$, it was only possible to collect 17 axial slices, thus excluding posterior parietal areas, dorsal prefrontal areas, and the most ventral part of the cerebellum from the imaging volume. Prior to the functional runs, 17 anatomical, T1-weighted, modified driven-equilibrium Fourier-transform images (Norris, 2000; Uğurbil et al., 1993) were acquired (data matrix $256 \times 256$ voxels, TR $1.3 \mathrm{sec}$, TE $10 \mathrm{msec}$ ), as well as $17 \mathrm{~T} 1$-weighted EPI images with the same spatial orientation as the functional data. The latter were used to co-register the functional scans with previously acquired high-resolution full-brain 3-D scans.

\section{Data Analysis}

The MRI data were processed using the software package LIPSIA (Lohmann et al., 2001). Functional data were corrected for motion artifacts using a matching metric based on linear correlation. To correct for the temporal offset between the slices acquired in one scan, a cubic-spline interpolation was applied. A temporal high-pass filter with a cutoff frequency of $1 / 109 \mathrm{~Hz}$ was used for baseline correction of the signal, and a spatial Gaussian filter with a $5.65-\mathrm{mm}$ full width at half maximum (FWHM) was applied. The anatomical slices were co-registered with the full-brain scan that resided in the stereotaxic coordinate system and were transformed by linear scaling to a standard size (Talairach \& Tournoux, 1988). The transformation parameters obtained from this step were subsequently ap- plied to the functional slices, so that these slices were also registered within the stereotaxic space. This linear normalization process was improved by a subsequent processing step in which we performed an additional, nonlinear normalization (Thirion, 1998). Slice gaps were scaled using a trilinear interpolation, generating output data with a spatial resolution of $3 \times 3 \times 5 \mathrm{~mm}$ (i.e., $45 \mathrm{~mm}^{3}$ ).

The statistical evaluation was based on a least-squares estimation using the general linear model (GLM) for serially autocorrelated observations (random-effects model; Friston, 1994; Friston, Frith, Turner, \& Frackowiak, 1995; Worsley \& Friston, 1995). An event-related design was implemented - that is, the hemodynamic response function was modeled by means of the experimental conditions for each stimulus (where the "event" was the onset of stimulus presentation). The design matrix was generated utilizing a synthetic hemodynamic response function and its first and second derivatives (Friston et al., 1998), with a response delay of $6 \mathrm{sec}$. The model equation, including the observation data, the design matrix, and the error term, was convolved with a Gaussian kernel of dispersion of 4-sec FWHM to deal with the temporal autocorrelation (Worsley \& Friston, 1995). Contrast images - that is, estimates of the raw-score differences between specified conditions - were generated for each participant. The single-participant contrast images were entered into a second-level analysis based on Bayesian statistics (Neumann \& Lohmann, 2003). In the approach of Neumann and Lohmann, both posterior probability maps and maps of the sizes of the effects of interest within groups of participants are calculated on the basis of the resulting least-squares estimates of the parameters of the GLM. The output of this Bayesian second-level analysis was a map showing probabilities that the contrast was larger than zero. For visualization, a threshold of $99.9 \%$ was applied to these probability maps.

The reasons to use a Bayesian second-level analysis with fMRI data are manifold: Comparison between traditional analysis, based on $t$ statistics, and Bayesian second-level analysis has shown that the latter is more robust against outliers. Furthermore, the Bayesian approach overcomes some problems of null-hypothesis significance testing, such as the need to correct for multiple comparisons, as well as providing estimates for the size of an effect of interest and for the probability that the effect actually occurs in the population (Neumann \& Lohmann, 2003). That is because the use of Bayesian statistics precludes the need to correct for false positives, because no test is performed in which an initial hypothesis could be falsely rejected. Rather, the probability of a contrast is calculated directly from our model parameters ( $\beta$ values).

\section{RESULTS}

\section{Behavioral Results}

Participants responded on average to $97.2 \%$ ( $S E 1.2)$ of the trials. They categorized $57.8 \%$ ( $S E 3.2)$ of the trials as coherent - that is, featuring sound stimuli that represented meaningful auditory events. In all, 74\% (SE 2.7) of the forward-played sound stimuli were judged to be coherent (hits), and the remaining 26\% (SE 2.7) of those stimuli were incorrectly classified as noncoherent (misses). In addition, $59 \%$ ( $S E 4.1$ ) of the backward-played sound stimuli were correctly classified as noncoherent (correct rejections [CRs]), whereas $41 \%$ (SE 4.1) were incorrectly classified as coherent (FAs). In line with the signal detection theory (SDT) approach, two parameters were estimated from the experimental data: the strength of the signal $\left(d^{\prime}\right)$ and the strategy of response of participants ( $C$, as well as a variant of $C$ called $\beta$; see Abdi, 2007). With $d^{\prime}=.87$, the mean of the signal distribution was almost one standard deviation away from the mean of the noise distribution, indicating that the task was relatively 
difficult. One way to express the location of participants' response thresholds is in relation to what is called the ideal observer, who conjointly minimizes the probabilities of a miss and an FA. In the present experiment, the ideal observer was characterized by a $d^{\prime}$ of .44 , an estimation of $C$ of -.21 , and a $\beta$ of .84. That is, our participants were obviously, but not perfectly, discriminating between coherent and noncoherent sound stimuli (as indicated by their $d^{\prime}=.87$ ), and they showed a liberal response strategy - that is, in case of doubt, they would rather say that an auditory signal implied coherence than that it did not. This response pattern, which ensures hits (successful recognition) at the cost of increased FAs (failure to reject), emphasizes a motivational orientation toward a relatively risky processing style in which alternatives are actively sought (see Crowe \& Higgins, 1997).

Over the course of the experimental session, mean reaction times (RTs, in milliseconds) differed significantly between the four response categories $[F(3,11)=49, p<$ $.0001]$; that is, participants responded significantly faster on trials they judged to be coherent $(M \pm S E: 2,453 \pm 118)$ than on those they judged to be noncoherent $(3,242 \pm 138)$ $[t(13)=-7.58, p<.0001]$. With regard to the latter trials, a post hoc test for misses and CRs revealed no significant RT differences $[t(13)=0.76, p=.46]$, yet the post hoc test for hits and false alarms showed that participants responded significantly faster on hits $(2,240 \pm 127)$ than on FAs $(2,665 \pm 114)[t(13)=-8.14, p<.0001]$. Hence, irrespective of the assignments of fingers to button responses, the group-averaged RT to the sound stimuli judged to be coherent was $2.5 \mathrm{sec}$, as compared with $3.2 \mathrm{sec}$ to those judged to be noncoherent. The significantly longer RTs for "noncoherent" judgments may imply that greater processing demands were required when judging sound stimuli to be unrecognizable. Consequently, this suggests that those regions preferentially engaged by the recognizable environmental sounds were not simply modulated by an overall greater task difficulty or task demands. A comparison of the forward-played $(2,493 \pm 133)$ and backward-played $(2,998 \pm 129)$ sound stimuli revealed a significant RT difference $[t(13)=$ $-11.73, p<.0001$ ], in that the forward-played stimuli were responded to more quickly, as well as a significant difference in the rates of correct responses $[t(13)=2.34$, $p<.036]$. That is, the forward-played stimuli elicited correct responses in $74 \%$ of the trials, whereas only $59 \%$ of the backward-played stimuli were correctly identified as noncoherent. With regard to the four different versions of the stimuli - that is, no distortion, superposition with pink noise, fence distortion, and fence distortion with superimposed pink noise - significant differences were observed in neither RTs $[F(3,11)=0.35, p=.63]$ nor percentages correct $[F(3,11)=1.82, p=.18]$.

After the experimental session, only those sound stimuli that participants categorized as coherent were played to them again, and they were asked to name each stimulus if they could. In all, $74 \%$ of those stimuli could be correctly named (median $80 \%$, range $42 \%-90 \%$ ); the rest were either unknown $(13 \%)$ or associated with a feeling of familiarity (13\%).
The questionnaire capturing the preference for intuition versus deliberation (PID; Betsch, 2004) revealed a dominant preference for intuition in 6 participants and a preference for deliberation in the rest. None of the correlational analyses of the percentages of signal changes with PID turned out to be significant. Hence, the general preference for intuition or deliberation in decision-making, as indicated by the PID, seems not to have governed or influenced the processing of auditory coherence judgments.

\section{Imaging Results}

To reveal brain regions particularly involved in the processing of intuitive judgments, trials for sound stimuli incorrectly categorized as coherent (FAs) were compared with those for stimuli correctly categorized as coherent (hits). Since both types of stimuli were answered to with "yes," confounding effects due to response mode were excluded. As expected, activation was revealed within the left rostral medial OFC, lateral OFC, right frontal operculum (FOP), inferior frontal gyrus, left anterior medial temporal pole, and middle temporal gyrus (MTG), as well as bilaterally within the superior temporal gyrus (see Table 1 and Figure 1A). The reverse contrast (i.e., hits vs. FAs) did not reveal any activation foci in the OFC.

Although the backward- and forward-played data sets were ideally matched for overall duration, intensity, spectral variation, spectral content, and acoustic complexity, they differed in that the backward-played sound stimuli (FAs and CRs) were distorted in the temporal domain, thereby altering the temporal phase and onset of events. Accordingly, one could argue that the activation in the contrast of FAs versus hits reflects differences in the temporal acoustic properties of the backward- versus the forwardplayed sound stimuli, rather than differences in intuitive recognition of these stimuli per se. However, when we exclusively compared the backward-played sound stimuli, whether categorized as coherent (FAs) or noncoherent (CRs), we still found significant activation within the left rostral medial OFC $(x, y, z:-17,51,-15)$. In addition, significant activation was observed within the left MTG $(-56,-39,-6)$, inferior prefrontal gyrus $(-47,30,6)$, and superior temporal gyrus $(-56,9,0)$. This result suggests that OFC activation reflects intuitive recognition rather than stimulus differences. Yet the reliability of this proposition is limited by the fact that FAs and CRs differ in response mode ("yes" vs. "no").

To overcome the possibly confounding effects of response mode and temporal phase, two further analyses were indicated: (1) an interaction contrast of the two factors stimulus-dependent splitting of trials (objectively coherent/noncoherent) and performance-dependent splitting (subjectively coherent/noncoherent), as well as (2) a conjunction analysis of all three possible direct contrasts with FAs - that is, FAs versus hits, CRs, and misses. The latter calculation was implemented because direct contrasts between FAs and the other three response categories would have suffered from unequal numbers of trials. The interaction contrast revealed significant activation within the left rostral medial OFC for objectively incorrect responses (FAs and misses; see Table 1 and Figure 1B). 
Table 1

Laterality, Anatomical Specification, and Talairach Coordinates $(x, y, z)$ for Activation According to the Second-Level Bayesian Analysis, for Direct Contrasts and Conjunction Analyses

\begin{tabular}{ccccc} 
Analysis, for Direct Contrasts and Conjunction Analyses & \\
\hline Area & $x$ & $y$ & $z$ \\
\hline
\end{tabular}

False Alarms $>$ Hits

L. rostral medial orbitofrontal cortex (OFC)

L. lateral OFC

$\begin{array}{lll}-17 & 51 & -12\end{array}$

$-50 \quad 39$

. inferior frontal gyrus

$-50$

R. inferior frontal gyrus

$37 \quad 33$

R. frontal operculum (FOP)/anterior insula

37

40

$-56$

L. superior temporal gyrus (STG)

R. STG

L. anteromedial temporal pole

L. middle temporal gyrus (MTG)

Interaction Contrast

(Stimulus- and Performance-Dependent Splitting)

$\begin{array}{lrrr}\text { L. rostral medial OFC } & -20 & 48 & -12 \\ \text { R. anterior inferior frontal gyrus } & 43 & 42 & 3 \\ \text { R. inferior frontal gyrus } & 34 & 39 & 15 \\ \text { R. FOP/anterior insula } & 43 & 21 & -3\end{array}$

Conjunction of Three Contrasts of FAs

L. rostral medial OFC $-17 \quad 52$

L. lateral OFC

Ventromedial prefrontal cortex

$-47$

R. FOP/anterior insula

R. inferior frontal gyrus

L. STG

R. STG

L. anteromedial temporal pole

L. MTG

Group Comparison High > Low Responders (Postsession Questionnaire)

L. rostral OFC

L. central OFC

R. central OFC

L. FOP/anterior insula

R. FOP/anterior insula

R. insular cortex

R. anterior temporal lobe

Conjunction Auditory and Visual Experiment

L. rostral medial OFC

R. FOP/anterior insula

L. MTG

$\begin{array}{rr}-21 & 48 \\ 43 & 15 \\ -50 & -52\end{array}$

Forward-Played Stimuli $>$ Backward-Played Stimuli

L. inferior frontal gyrus

R. inferior temporal gyrus

L. temporoparietal junction area

R. temporoparietal junction area

$\begin{array}{rrr}-47 & 9 & 21 \\ 46 & -51 & -6 \\ -38 & -57 & 18 \\ 40 & -69 & 24\end{array}$

Note-All activation foci were set to posterior probabilities of $99.9 \%$.

Furthermore, activation was observed within the right inferior frontal gyrus and FOP/anterior insula. To test which factor was driving this effect, we calculated percentage signal changes within the left rostral medial OFC $(-20$, $48,-12)$; the results revealed FAs to be the driving factor for OFC activation (see Figure 1F). Likewise, significant activation within the rostral medial and lateral OFC was observed for the conjunction analysis of the three FA contrasts, as well as activation within the ventromedial prefrontal cortex, right FOP/anterior insula, inferior frontal gyrus, left MTG, and anteromedial temporal pole, and bilateral activation within the superior temporal gyrus (see Table 1 and Figure 1C).
According to our assumption that participants could be considered more intuitive if, in the postsession questioning, they provided many ideas about the stimuli they had previously designated as coherent, we calculated a median split for the contrast of FAs versus hits. The results revealed activation within the left rostral OFC, bilaterally within the central OFC and the FOP/anterior insula, and within the right anterior temporal lobe for the group who had generated many ideas (see Table 1 and Figure 1D).

If activation within the OFC simply reflects the monitoring of reward value of the coherent sound stimuli, activation within this area should also be elicited by forward-played sound stimuli, irrespective of a participant's response. Yet the resulting pattern of activation for the contrast of forward- versus backward-played sound stimuli involved three foci excluding the OFC: Significant activation was found within the left inferior prefrontal cortex, within the right inferior temporal gyrus, and within the left posterior temporal gyrus near the junction with the parietal lobe (see Table 1). Thus, we may exclude the possibility that activation in the OFC simply reflects the monitoring of reward value.

The present study was aimed at investigating the multimodality of OFC activation during intuitive judgment processes. Hence, a conjunction analysis with our previous study was suggested - that is, a test for a logical AND, so as to identify those brain regions that are commonly activated by intuitive judgment processes in both auditory and visual coherence tasks (see Nichols, Brett, Andersson, Wager, \& Poline, 2005; Volz \& von Cramon, 2006). The conjunction between the contrast from our visual study (coherent $>$ noncoherent trials) and the contrast FAs versus hits from the present, auditory study revealed significant activation within the left rostral medial OFC, left MTG, and right FOP/anterior insula (see Table 1 and Figure 1E).

\section{DISCUSSION}

In the present fMRI study, we attempted to examine the neural substrates of coherence judgments in the context of auditory discovery, which we comprehended as reflecting intuitive thinking processes. Specifically, according to the learning perspective, we conceived of intuitions as difficult-to-articulate, affect-laden recognitions or judgments, which are based on prior learning and experience (see Sadler-Smith, 2008). Particularly, we proposed that implicit extracted clues of coherence activate a relevant mnemonic network, which integrates the entire stream of prior experiences that make up the gist of the entity (also denoted as a chunk). In the present forced choice paradigm, participants were presented with either short sequences of nonverbal environmental sounds or temporally reversed versions of the same sounds, both of which were superimposed with pink noise and/or with periods of zero sound energy. While listening, participants performed a sound categorization task; that is, they had to indicate as fast as possible, for each sound, whether or not it was coherent. This ability to recognize different types of environmental (nonverbal) sounds is thought 
A

FAs vs. Hits

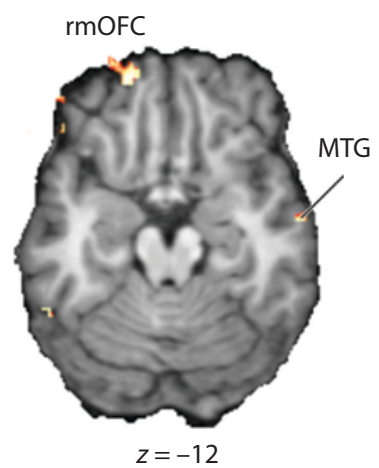

C Conjunction FA Contrasts

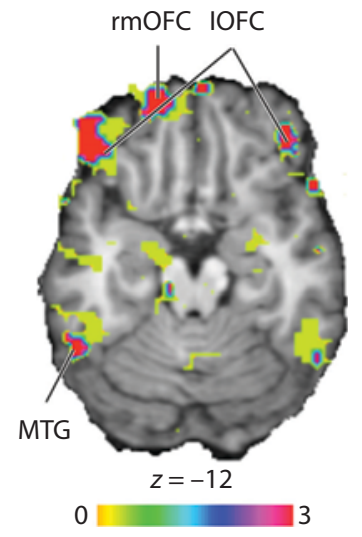

E Conjunction With Visual Exp.

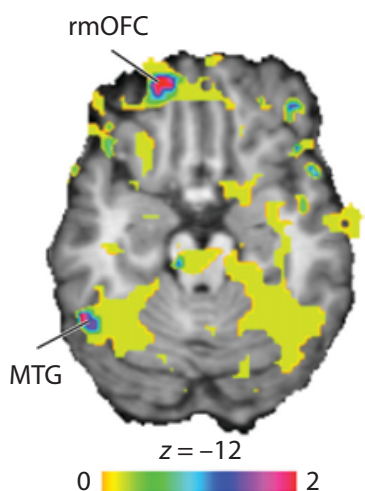

B Interaction Contrast

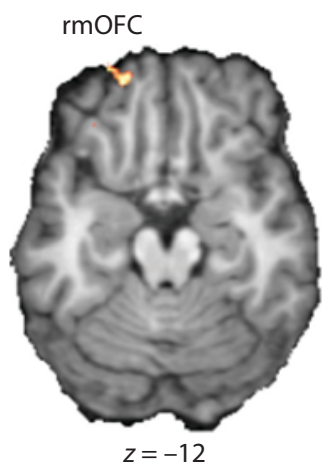

D

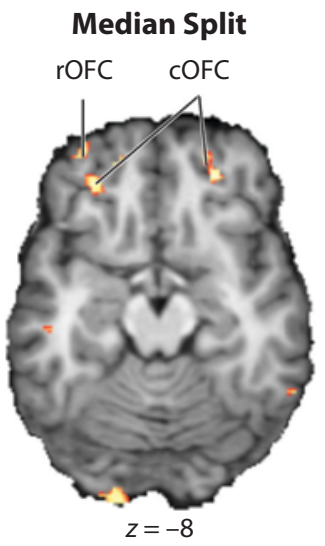

F Signal Change in rmOFC (\%)

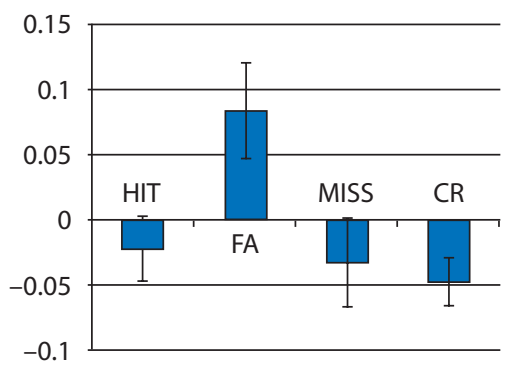

Figure 1. Group-averaged activations are shown on axial slices taken from an individual brain normalized and aligned to the Talairach and Tournoux (1988) stereotaxic space. (A) Direct contrast between trials participants incorrectly judged to be coherent (false alarms, or FAs) and trials they correctly judged to be coherent (hits). (B) Interaction contrast between objectively and subjectively coherent/noncoherent trials (i.e., stimulus- and performance-dependent splitting of trials). (C) Conjunction analysis of all possible FA contrasts. (D) Results of the median split for the contrast of FAs versus hits for the group who provided many solutions in the postsession questionnaire, given trial stimuli they had previously indicated as coherent. (E) Conjunction analysis of auditory (see panel $A$ ) and visual coherence judgments. The contrast in the latter modality was taken from a previous study on visual coherence judgments (Volz \& von Cramon, 2006). (F) Percentage of signal change within the rostral medial OFC $(x=$ $-20, y=48, z=-12)$. cOFC, central orbitofrontal cortex; IOFC, lateral orbitofrontal cortex; MTG, middle temporal gyrus; rmOFC, rostral medial orbitofrontal cortex; rOFC, rostral orbitofrontal cortex. 
to be accomplished in part by automatically abstracting the physical properties of a sound and matching them to the implicit known characteristics of given sound categories. Since forward-played sound stimuli were likely to be correctly classified as coherent on the basis of fully formed judgments, we conceived that FAs could in part reflect intuitive judgment processes, because the temporally reversed sounds matched the physical features of the original coherent sounds in all respects except their temporal sequence. Accordingly, the majority of the physical features of the backward sound stimuli could activate a chunk. We chose to apply a distortion manipulation so as to encourage participants to (implicitly) evaluate the various acoustical features of the stimuli other than their temporal sequence.

The present imaging results revealed rostral medial OFC activation that was likely specific to intuitive coherence judgments in the auditory domain. Furthermore, a conjunction analysis between the present results and those from a previous study on visual intuitive coherence judgments (Volz \& von Cramon, 2006) revealed that the rostral medial OFC is a common neural substrate for coherence. Hence, we conclude that, in both the visual and auditory modalities during intuitive coherence judgments, the OFC is likely to subserve the detection of potential content from coarse aspects of the sensory input. All in all, on the basis of the present data and of previous studies on object recognition (Bar, 2007; Bar et al., 2006), we here propose a very preliminary neurocognitive model of intuitive judgments in the auditory domain.

The OFC is said to be involved in sensory integration, emotion regulation, and the representation of the affective value of reinforcers (see Arana et al., 2003; Bechara, H. Damasio, \& A. R. Damasio, 2000; Gottfried, O'Doherty, \& Dolan, 2003; Kringelbach, 2005; Kringelbach \& Rolls, 2004; O’Doherty, Deichmann, Critchley, \& Dolan, 2002). These functional accounts stem from human fMRI and lesion studies. Specification of the OFC's function derives mainly from animal studies (see Boulougouris, Dalley, \& Robbins, 2007; Roesch \& Schoenbaum, 2006; Saddoris, Gallagher, \& Schoenbaum, 2005; Schoenbaum \& Roesch, 2005; Schoenbaum et al., 2006): Single-cell recordings in rats and monkeys have revealed that, early in learning, neurons in the OFC will respond to one outcome but not to another-for instance, when a rat learns to discriminate between two odors on the basis of the outcomes they predict (e.g., sucrose or quinine solution). In this case, the neurons begin to respond in anticipation of their preferred outcome. After learning, the neurons come to be already activated by the cues that predict the preferred outcome, thereby signaling the expected outcome even before a response is made. From their study, Schoenbaum et al. concluded that the selective activity within the OFC represents the expectation of an individual on the basis of its experience of likely outcomes. These results nicely fit with our assumption that the OFC rapidly detects potential content by serving as a repository of current associations between particular cues and outcomes, given the current circumstances. During daily experience, in- dividuals learn that specific abstracted cues are associated with preferred outcomes, so that a "probabilistic matrix" evolves. Most of this knowledge is supposed to be implicit and to depend on the amount and the timing of experience with the specific cue-outcome relations (see Bar, 2007). This notion is compatible with the idea that experts develop an intuitive understanding within their field of expertise through the acquisition of a large number of chunks associated with the relevant action knowledge. Recently, Raab and Johnson (2007) specified the locus of this expertise effect in the accessibility and quality of an initial solution, rather than in the subsequent deliberation. That is, the readout of a specific mnemonic network seems to be more decisive than it would be following strategic effects. An interesting future object of investigation would be a comparison of OFC activation between experts and novices.

The task in the present auditory paradigm was chosen as a problem at which we are all experts - namely, the detection of coherent meaningful sounds in the environment. This task would not, per se, necessarily require individuals to rely on affect-laden recognition judgments. Yet the distortion manipulations we applied, such as the lack of reliable auditory information and of feedback, suggest that an intuitive approach would be needed. Accordingly, to perform successfully despite the difficulties, individuals probably had to rely on prior learning and experiences, which subsequently biased their thoughts and actions. It has been suggested that this biasing signal arises in the OFC and that it is experienced as an initial guess and embodied in a "gut feeling," both of which suggest a specific course of action. The notion of a biasing somatic signal is consistent with the findings of A. R. Damasio and colleagues using the Iowa gambling task with both healthy participants and OFC-damaged patients (see, e.g., Bechara, A. R. Damasio, H. Damasio, \& Anderson, 1994; Bechara, Tranel, \& Damasio, 2002; Damasio, 1994). In this task, participants choose cards either with high rewards and occasional large penalties or with low rewards but no penalties. Healthy participants initially choose from the high-reward deck but later bias their choices to the low-reward deck. At first, this change in behavior cannot consciously be justified; instead, participants report basing their choices on a mere feeling until the penalties become apparent. Research has suggested that this "gut" feeling is reflected in anticipatory skin conductance response (SCR) activity in response to a possible outcome of the action (e.g., Bechara et al., 2000). OFC-damaged patients also prefer the high-reward deck at the beginning, but they fail to modify their responding; it has been suggested that this pattern reflects an inability to generate the outcome expectancies necessary to compare the relative values of the preferred and unpreferred outcomes (Schoenbaum $\&$ Roesch, 2005). Interestingly, these patients also fail to generate anticipatory SCRs before picking a card (see, e.g., Bechara et al., 2002).

In general, we assume that whenever situational constraints preclude flawless processing, individuals may rely less on an analytic, rule-based, and explicit process- 
ing mode than on an associative, holistic, and implicit one, so as to maintain flexibility and freedom of action (see Stanovich \& West, 2000). The latter mode of processing can provide a quick and low-effort evaluation of the stimulus or set of cues by relying on associative information, particularly on information about the value of likely outcomes. As we have outlined above, research has suggested that the OFC plays a crucial role in the generation and use of outcome expectancies (see Roesch \& Schoenbaum, 2006). Accordingly, when a situation requires tapping into implicit knowledge, we suggest that freedom of action is preserved by the OFC's detecting potential content and subsequently biasing thought, inquiry, and action. Possibly, this guiding signal is conveyed as an affective reaction (somatic signal) manifested in anticipatory SCRs.

From the results of the contrast of FAs versus hits and the conjunction of all three FA contrasts, we further conclude that whenever participants (incorrectly) indicated coherence, they had indeed "heard" the putative sound source and had aimed at detecting the salient event boundaries. We assume this because of activation within the MTG, FOP, and right inferior frontal gyrus. The MTG has been shown to be crucial for the recognition of familiar environmental sound sources and to be implicated in the retrieval of action knowledge - that is, of how a sound is likely to have been produced (see Lewis, Brefczynski, Phinney, Janik, \& DeYoe, 2005; Lewis et al., 2004). The right ventrolateral prefrontal cortex and FOP have repeatedly been found to be implicated in the detection of violations in musical predictions (e.g., Koelsch et al., 2002; Maess, Koelsch, Gunter, \& Friederici, 2001). More specifically, this activation is critical for the perceptual segmentation of salient, coarse-grained event boundaries (Sridharan, Levitin, Chafe, Berger, \& Menon, 2007). Accordingly, in the present paradigm, we suggest that participants try to retrieve action knowledge so as to downsize the number of possible auditory sound sources. Subsequently, a selection within this reduced space has to be accomplished - that is, via the detection of salient events (boundaries) on the basis of the sensory features of the stimulus stream.

After taking into account the results of Bar et al. (2006), the finding of OFC activation in difficult recognition tasks in the auditory and visual modalities (Volz \& von Cramon, 2006), and the data on cortico-cortical connections (see Barbas, 1992; Seltzer \& Pandya, 1989), we propose the following preliminary neurocognitive model of intuitive judgments: A partially analyzed version of the input (in the present case, a noisy auditory signal) is quickly projected from auditory areas to the OFC (Kringelbach \& Rolls, 2004; Pandya \& Yeterian, 1985). In line with tracing studies in the rhesus monkey, various researchers have suggested that the OFC receives input both from multimodal convergence zones in the superior temporal sulcus and from auditory association areas in the anterior half of the superior temporal gyrus (Barbas, 1992; Barbas et al., 2005; Pandya \& Yeterian, 1985; Seltzer \& Pandya, 1989). These connections, it should be noted, are known to be reciprocal (Pandya \& Yeterian, 1985). The analysis of the coarse auditory input is supposed to occur in parallel with the more systematic, and probably slower, propagation of the same information along organized auditory processing pathways, involving secondary and tertiary auditory processing domains in the superior temporal gyrus and extending up to the junction with the parietal lobe (Kaas \& Hackett, 2000). The coarse representation of the input, we suggest, activates specific outcome expectancies within the rostral OFC, which in turn may activate the most probable interpretation of the input in recognition-related regions within the temporal cortex. By combining the initial guess from the OFC, based on the fuzzy input, with the output of the more systematic analysis, recognition is supported by confining the number of possible object representations.

\section{AUTHOR NOTE}

The authors thank Ricarda I. Schubotz for helpful comments on the final draft of this article, Eva Weig for developing the stimulus materials and for assistance in the data collection, Kerstin Flake for support concerning graphical matters, and Tracy Farr for help with proofreading. Correspondence related to this article may be sent to K. G. Volz, Max Planck Institute for Neurological Research, P.O. Box 4106 29, D-50866 Cologne, Germany (e-mail: volz@nf.mpg.de).

\section{REFERENCES}

AbDI, H. (2007). Signal detection theory (SDT). In N. J. Salkind (Ed.), Encyclopedia of measurement and statistics. Thousand Oaks, CA: Sage.

ANDERSON, J. R. (1983). A spreading activation theory of memory. Journal of Verbal Learning \& Verbal Behavior, 22, 261-295.

Arana, F. S., Parkinson, J. A., Hinton, E., Holland, A. J., Owen, A. M., \& Roberts, A. C. (2003). Dissociable contributions of the human amygdala and orbitofrontal cortex to incentive motivation and goal selection. Journal of Neuroscience, 23, 9632-9638.

BAR, M. (2007). The proactive brain: Using analogies and associations to generate predictions. Trends in Cognitive Sciences, 11, 280-289.

Bar, M., Kassam, K. S., Ghuman, A. S., Boshyan, J., Schmid, A. M., DAle, A. M., ET AL. (2006). Top-down facilitation of visual recognition. Proceedings of the National Academy of Sciences, 103, 449-454.

BARBAS, H. (1992). Architecture and cortical connections of the prefrontal cortex in the rhesus monkey. Advances in Neurology, 57, 91-115.

Barbas, H., Medalla, M., Alade, O., Suski, J., Zikopoulos, B., \& LERA, P. (2005). Relationship of prefrontal connections to inhibitory systems in superior temporal areas in the rhesus monkey. Cerebral Cortex, 15, 1356-1370.

Bechara, A., Damasio, A. R., Damasio, H., \& Anderson, S. W. (1994). Insensitivity to future consequences following damage to human prefrontal cortex. Cognition, 50, 7-15.

Bechara, A., Damasio, H., \& Damasio, A. R. (2000). Emotion, decision making and the orbitofrontal cortex. Cerebral Cortex, 10, 295-307.

Bechara, A., Tranel, D., \& Damasio, A. R. (2002). The somatic marker hypothesis and decision-making. In F. Boller \& J. Grafman (Series Eds.), J. Grafman (Vol. Ed.), Handbook of neuropsychology (2nd ed.; Vol. 7, pp. 117-143). Amsterdam: Elsevier.

Betsch, C. (2004). Präferenz für Intuition und Deliberation: Inventar zur Erfassung von affekt- und kognitionsbasiertem Entscheiden. Zeitschrift für Differentielle \& Diagnostische Psychologie, 25, 179-197.

Birn, R. M., Cox, R. W., \& Bandettini, P. A. (2002). Detection versus estimation in event-related fMRI: Choosing the optimal stimulus timing. NeuroImage, 15, 252-264.

Boulougouris, V., Dalley, J. W., \& Robbins, T. W. (2007). Effects of orbitofrontal, infralimbic and prelimbic cortical lesions on serial spatial reversal learning in the rat. Behavioural Brain Research, 179, 219-228. 
Bowers, K. S., Regehr, G., Balthazard, C., \& Parker, K. (1990). Intuition in the context of discovery. Cognitive Psychology, 22, 72-110.

Crowe, E., \& Higgins, E. T. (1997). Regulatory focus and strategic inclinations: Promotion and prevention in decision-making. Organizational Behavior \& Human Decision Processes, 69, 117-132.

Damasio, A. R. (1994). Descartes' error: Emotion, reason, and the human brain. New York: Putnam.

EPSTEIn, S. (1973). The self-concept revisited, or a theory of a theory. American Psychologist, 28, 404-416.

FRISTON, K. J. (1994). Functional and effective connectivity in neuroimaging: A synthesis. Human Brain Mapping, 2, 56-78.

Friston, K. J., Fletcher, P., Josephs, O., Holmes, A., RugG, M. D., \& TURNER, R. (1998). Event-related fMRI: Characterizing differential responses. Neurolmage, 7, 30-40.

Friston, K. J., Frith, C. D., Turner, R., \& Frackowiak, R. S. (1995). Characterizing evoked hemodynamics with fMRI. NeuroImage, 2 , 157-165.

Gilovich, T., Griffin, D., \& Kahneman, D. (Eds.) (2002). Heuristics and biases: The psychology of intuitive judgement. Cambridge: Cambridge University Press.

Gottrried, J. A., O’Doherty, J., \& Dolan, R. J. (2003). Encoding predictive reward value in human amygdala and orbitofrontal cortex. Science, 301, 1104-1107.

HaMmond, K. R. (2007). Beyond rationality: The search for wisdom in a troubled time. Oxford: Oxford University Press.

Hammond, K. R., Hamm, R. M., Grassia, J., \& Pearson, T. (1987). Direct comparison of the efficacy of intuitive and analytical cognition in expert judgment. IEEE Transactions on Systems, Man, \& Cybernetics, 17, 753-770.

Hogarth, R. M. (2001). Educating intuition. Chicago: University of Chicago Press.

Ilg, R., Vogeley, K., Goschke, T., Bolte, A., Shah, J. N., Pöppel, E., \& FINK, G. R. (2007). Neural processes underlying intuitive coherence judgments as revealed by fMRI on a semantic judgment task. NeuroImage, 38, 228-238.

JeZZard, P., \& Balaban, R. S. (1995). Correction for geometric distortion in echo planar images from B0 field variations. Magnetic Resonance in Medicine, 34, 65-73.

KAas, J. H., \& HacKetT, T. A. (2000). Subdivisions of auditory cortex and processing streams in primates. Proceedings of the National Academy of Sciences, 97, 11793-11799.

Kahneman, D., \& FredericK, S. (2002). Representativeness revisited: Attribute substitution in intuitive judgment. In T. Gilovich, D. Griffin, \& D. Kahneman (Eds.), Heuristics and biases: The psychology of intuitive judgement (pp. 49-81). Cambridge: Cambridge University Press.

Kahneman, D., Slovic, P., \& Tversky, A. (Eds.) (1982). Judgment under uncertainty: Heuristics and biases. Cambridge: Cambridge University Press.

Koelsch, S., Gunter, T. C., von Cramon, D. Y., Zysset, S., LohMANN, G., \& Friederici, A. D. (2002). Bach speaks: A cortical "language-network" serves the processing of music. NeuroImage, 17, 956-966.

Kringelbach, M. L. (2005). The human orbitofrontal cortex: Linking reward to hedonic experience. Nature Reviews Neuroscience, $\mathbf{6}$, 691-702.

KringelbaCh, M. L., \& Rolls, E. T. (2004). The functional neuroanatomy of the human orbitofrontal cortex: Evidence from neuroimaging and neuropsychology. Progress in Neurobiology, 72, 341-372.

Lewis, J. W., Brefczynski, J. A., Phinney, R. E., Janik, J. J., \& DeYoe, E. A. (2005). Distinct cortical pathways for processing tool versus animal sounds. Journal of Neuroscience, 25, 5148-5158.

Lewis, J. W., Wightman, F. L., Brefczynski, J. A., Phinney, R. E., BINDER, J. R., \& DeYoe, E. A. (2004). Human brain regions involved in recognizing environmental sounds. Cerebral Cortex, 14, 10081021.

Lohmann, G., Müller, K., Bosch, V., Mentzel, H., Hessler, S., Chen, L., ET AL. (2001). LIPSIA-A new software system for the evaluation of functional magnetic resonance images of the human brain. Computerized Medical Imaging \& Graphics, 25, 449-457.

Maess, B., Koelsch, S., Gunter, T. C., \& Friederici, A. D. (2001) Musical syntax is processed in Broca's area: An MEG study. Nature Neuroscience, 4, 540-545.
Medin, D. L., \& Coley, J. D. (1998). Concepts and categorization. In J. Hochberg (Ed.), Perception and cognition at century's end: History, philosophy, theory (Handbook of Perception and Cognition, 2nd ed., pp. 403-440). San Diego: Academic Press.

Miezin, F. M., Maccotta, L., Ollinger, J. M., Petersen, S. E., \& BUCKNER, R. L. (2000). Characterizing the hemodynamic response: Effects of presentation rate, sampling procedure, and the possibility of ordering brain activity based on relative timing. NeuroImage, 11, 735-759.

Neumann, J., \& Lohmann, G. (2003). Bayesian second-level analysis of functional magnetic resonance images. NeuroImage, 20, 13461355.

Nichols, T., Brett, M., Andersson, J., Wager, T., \& Poline, J. B. (2005). Valid conjunction inference with the minimum statistic. NeuroImage, 25, 653-660.

NorRIs, D. G. (2000). Reduced power multislice MDEFT imaging. Journal of Magnetic Resonance Imaging, 11, 445-451.

Norris, D. G., Zysset, S., Mildner, T., \& Wiggins, C. J. (2002). An investigation of the value of spin-echo-based fMRI using a Stroop color-word matching task and EPI at 3 T. Neurolmage, 15, 719-726.

O'Doherty, J. P., Deichmann, R., Critchley, H. D., \& Dolan, R. J. (2002). Neural responses during anticipation of a primary taste reward. Neuron, 33, 815-826.

Pandya, D. N., \& Yeterian, E. H. (1985). Architecture and connections of cortical association areas. In A. Peters \& E. G. Jones (Eds.), Cerebral cortex: Vol. 4. Association and auditory cortices (pp. 3-61). New York: Plenum.

Plessner, H., Betsch, C., \& Betsch, T. (Eds.) (2008). Intuition in judgment and decision making. Mahwah, NJ: Erlbaum.

RAaB, M., \& Johnson, J. G. (2007). Expertise-based differences in search and option-generation strategies. Journal of Experimental Psychology: Applied, 13, 158-170.

Reber, R., Wurtz, P., \& Zimmermann, T. D. (2004). Exploring "fringe" consciousness: The subjective experience of perceptual fluency and its objective bases. Consciousness \& Cognition, 13, 47-60.

Roesch, M., \& Schoenbaum, G. (2006). From associations to expectancies: Orbitofrontal cortex as gateway between the limbic system and representational memory. In D. H. Zald \& S. L. Rauch (Eds.), The orbitofrontal cortex (pp. 199-235). Oxford: Oxford University Press.

Saddoris, M. P., Gallagher, M., \& Schoenbaum, G. (2005). Rapid associative encoding in basolateral amygdala depends on connections with orbitofrontal cortex. Neuron, 46, 321-331.

SAdLeR-Smith, E. (2008). Inside intuition. London: Routledge.

Schmidt, C. F., Boesiger, P., \& Ishai, A. (2005). Comparison of fMRI activation as measured with gradient- and spin-echo EPI during visual perception. Neurolmage, 26, 852-859.

Schoenbaum, G., \& Roesch, M. [R.] (2005). Orbitofrontal cortex, associative learning, and expectancies. Neuron, 47, 633-636.

Schoenbaum, G., Roesch, M. R., \& Stalnaker, T. A. (2006). Orbitofrontal cortex, decision-making and drug addiction. Trends in Neurosciences, 29, 116-124.

Seltzer, B., \& Pandya, D. N. (1989). Frontal lobe connections of the superior temporal sulcus in the rhesus monkey. Journal of Comparative Neurology, 281, 97-113.

Sridharan, D., Levitin, D. J., Chafe, C. H., Berger, J., \& Menon, V. (2007). Neural dynamics of event segmentation in music: Converging evidence for dissociable ventral and dorsal networks. Neuron, $\mathbf{5 5}$, 521-532.

Stanovich, K. E., \& West, R. F. (2000). Individual differences in reasoning: Implications for the rationality debate? Behavioral \& Brain Sciences, 23, 645-665.

TAlairach, J., \& Tournoux, P. (1988). Co-planar stereotaxic atlas of the human brain: 3-dimensional proportional system. An approach to cerebral imaging. Stuttgart, Germany: Thieme.

THIRION, J. P. (1998). Image matching as a diffusion process: An analogy with Maxwell's demons. Medical Image Analysis, 2, 243-260.

Topolinski, S., \& Strack, F. (2007, September). Analysis or intuition? Analysis and intuition! The use of cognitive feelings in intuitive judgments. Paper presented at the 11th Meeting of the Social Psychology Section of the German Association of Psychology, Freiburg, Germany.

Topolinski, S., \& STRACK, F. (2008). Where there's a will-there's no 
intuition: The unintentional basis of semantic coherence judgments. Journal of Memory \& Language, 58, 1032-1048.

UĞurbil, K., Garwood, M., Ellermann, J., Hendrich, K., Hinke, R., Hu, X., ET AL. (1993). Imaging at high magnetic fields: Initial experiences at 4 T. Magnetic Resonance Quarterly, 9, 259-277.
Volz, K. G., \& von Cramon, D. Y. (2006). What neuroscience can tell about intuitive processes in the context of perceptual discovery. Journal of Cognitive Neuroscience, 18, 2077-2087.

Worsley, K. J., \& Friston, K. J. (1995). Analysis of fMRI time-series revisited-again. Neurolmage, 2, 173-181.

\section{APPENDIX}

Environmental Sounds

\begin{tabular}{lll}
\hline African drums & Apes & Banging of a door \\
Bell (3 versions) & Bell at a level crossing & Bell in a shop \\
Cowbell & Bells of Big Ben & Birds (2 versions) \\
Bouncing people & Breaking dishes (2 versions) & Breaking glass (5 versions) \\
Chicken clucking & Church bell (2 versions) & Clacking keys \\
Clanging metal & Clapping & Clock \\
Dishwasher & Doorbell & Drums (2 versions) \\
Eaves gutter & Fire & Gate creak \\
Glockenspiel (3 versions) & Hammering (2 versions) & Hammering on metal (2 versions) \\
Harp & Metronome & Piano \\
Pigs (2 versions) & Rattling coins & Rattling dishes \\
Ringing bell & Roasting meat & Rolling glass bottle \\
Rolling piece of metal (2 versions) & Running person & Ship's bell (3 versions) \\
Shooting (3 versions) & Shower (2 versions) & Splashing water \\
Table tennis & Triangle & Unscrewing a bottle \\
Washing dishes & Water gurgling in drain & Water in a washbasin (2 versions) \\
Water running & Whistling person & \\
\hline
\end{tabular}

(Manuscript received October 21, 2007;

revision accepted for publication April 18, 2008.) 